\title{
Diagnostic Performance of Optical Coherence Tomography Ganglion Cell-Inner Plexiform Layer Thickness Measurements in Early Glaucoma
}

Jean-Claude Mwanza, MD, PhD, ${ }^{1}$ Donald L. Budenz, MD, MPH, ${ }^{1}$ David G. Godfrey, MD, ${ }^{2}$ Arvind Neelakantan, MD, ${ }^{2}$ Fouad E. Sayyad, MD, ${ }^{3}$ Robert T. Chang, MD, ${ }^{4}$ Richard K. Lee, MD, PhD ${ }^{3}$

Purpose: To evaluate the glaucoma diagnostic performance of ganglion cell inner-plexiform layer (GCIPL) parameters used individually and in combination with retinal nerve fiber layer (RNFL) or optic nerve head $(\mathrm{ONH})$ parameters measured with Cirrus HD-OCT (Carl Zeiss Meditec, Inc, Dublin, CA).

Design: Prospective cross-sectional study.

Participants: Fifty patients with early perimetric glaucoma and 49 age-matched healthy subjects.

Methods: Three peripapillary RNFL and 3 macular GCIPL scans were obtained in 1 eye of each participant. A patient was considered glaucomatous if at least 2 of the 3 RNFL or GCIPL scans had the average or at least 1 sector measurement flagged at $1 \%$ to $5 \%$ or less than $1 \%$. The diagnostic performance was determined for each GCIPL, RNFL, and ONH parameter as well as for binary or-logic and and-logic combinations of GCIPL with RNFL or ONH parameters.

Main Outcome Measures: Sensitivity, specificity, positive likelihood ratio (PLR), and negative likelihood ratio (NLR).

Results: Among GCIPL parameters, the minimum had the best diagnostic performance (sensitivity, 82.0\%; specificity, 87.8\%; PLR, 6.69; and NLR, 0.21). Inferior quadrant was the best RNFL parameter (sensitivity, 74\%; specificity, 95.9\%; PLR, 18.13; and NLR, 0.27), as was rim area (sensitivity, 68\%; specificity, 98\%; PLR, 33.3; and NLR, 0.33) among ONH parameters. The or-logic combination of minimum GCIPL and average RNFL provided the overall best diagnostic performance (sensitivity, 94\%; specificity, 85.7\%; PRL, 6.58; and NLR, 0.07) as compared with the best RNFL, best ONH, and best and-logic combination (minimum GCIPL and inferior quadrant RNFL; sensitivity, 64\%; specificity, 100\%; PLR, infinity; and NPR, 0.36).

Conclusions: The binary or-logic combination of minimum GCIPL and average RNFL or rim area provides better diagnostic performances than those of and-logic combinations or best single GCIPL, RNFL, or ONH parameters. This finding may be clinically valuable for the diagnosis of early glaucoma. Ophthalmology 2014;121:849-854 @ 2014 by the American Academy of Ophthalmology.

Traditionally, optic disc examination by ophthalmoscopy, slit-lamp biomicroscopy, or optic disc photography have been the only methods available to clinicians for structural assessment in the diagnosis of glaucoma. However, clinical evaluation of the optic disc provides scant quantitative information, is subjective, and is characterized by interobserver variability. These limitations have stimulated over the years not only the identification of other structural parameters, but also the development of new automated imaging methods for their quantitative analysis. Various computerized imaging technologies that detect structural changes in the optic nerve head $(\mathrm{ONH})$, retinal nerve fiber layer (RNFL), ganglion cell complex, and ganglion cell-inner plexiform layer (GCIPL) have been introduced into clinical practice in recent years. These include the confocal scanning laser ophthalmoscopy, scanning laser polarimetry, and optical coherence tomography (OCT). Unlike the scanning laser ophthalmoscopy and scanning laser polarimetry, OCT measures the topography of the optic disc and differentiates between different retinal layers.

Although quantitative analysis of RNFL with OCT currently is the most widely used parameter for the diagnosis and monitoring of glaucoma, studies have also shown that analysis of $\mathrm{ONH}$ and ganglion cell layer parameters can be used for the same purpose. ${ }^{1-6}$ Recently, ganglion cell analysis (GCA) was developed as an additional tool for assessing structural change in glaucoma by detecting and measuring the thickness of the GCIPL. The GCA is based on the histologic observation that macular GCIPL is topographically less variable among normal individuals than RNFL and $\mathrm{ONH}^{7}$ which makes normal macular GCIPL parameters easier to identify and deviations from normal easier to detect and quantify. As a glaucoma diagnostic parameter, the discriminating ability of GCIPL is similar to that of RNFL and ONH based on comparison of area under the of the receiver operating characteristic curves. $^{4,8,9}$ All currently available OCT devices provide 
color-coded deviation, thickness, and significance maps that aid the clinician in determining whether the scanning results are within normal or outside normal range. We previously used the significance map to determine the sensitivity and specificity of RNFL measured with time-domain OCT (Carl Zeiss Meditec, Inc, Dublin, CA) ${ }^{10}$ and Cirrus HD-OCT (Carl Zeiss Meditec, Inc). ${ }^{10}$ In addition to RNFL, others have used the same strategy to study the sensitivity and specificity of ganglion cell complex and ONH with RTVue-100 (Optovue, Inc, Fremont, CA). ${ }^{12}$ Because measurements generated by various OCT devices are not interchangeable, it is important that diagnostic measures be evaluated for each device. The purpose of this study was to evaluate the diagnostic performance of GCIPL parameters used individually and in combination with retinal RNFL or ONH parameters measured with Cirrus HD-OCT.

\section{Methods}

\section{Subject Selection}

Subjects included in this study were recruited at the Bascom Palmer Eye Institute in Miami, Florida; the Glaucoma Associates of Texas in Dallas, Texas; and Stanford University in Palo Alto, California. Each center had the study protocol approved by its institutional review board before subject enrollment. All subjects were 18 years of age or older and signed an informed consent and Health Insurance Portability and Accountability Act forms. Patients with documented glaucoma defined as typical ONH cupping associated with glaucomatous visual field deficits on SITA standard 24-2 Humphrey visual field (Carl Zeiss Meditec, Inc) testing, with or without elevated intraocular pressure, were invited to participate. The diagnosis of early glaucoma was based on a visual field mean deviation of $-6 \mathrm{~dB}$ or more. ${ }^{13}$ Both glaucoma patients and normal subjects were included if they had a best-corrected visual acuity of $20 / 40$ or better and refractive error of between -5 and +5 diopters (D) in sphere or $2.5 \mathrm{D}$ or less in cylinder. Additional criteria for normal subjects (visual field not performed) were intraocular pressure of $21 \mathrm{mmHg}$ or less, normallooking $\mathrm{ONH}$ on ophthalmoscopy without cupping, asymmetry in cup-to-disc ratio of less than 0.2 , notching, or disc hemorrhage. Exclusion criteria for both groups of subjects included media opacities, nonglaucomatous optic neuropathy (i.e., multiple sclerosis, trauma), past or current retinal disease (i.e., retinal detachment, diabetic or infectious retinopathy, age-related macular degeneration), history of retinal surgery, laser or radiation therapy, or systemic medication that may induce optic neuropathy. Because of the age-related decline in RNFL and GCIPL thicknesses, recruitment was performed so as to age match each glaucoma patient to 1 normal subject within 5 years of age. None of the normal subjects included in this study were among those used to determine normative values for Cirrus HD-OCT RNFL, ONH, or GCIPL. If both eyes qualified, only 1 eye randomly selected by each site's principal investigator was included in the study.

\section{Optical Coherence Tomography Scanning Procedure}

After pupil dilation, each qualifying eye underwent 3 sessions of peripapillary scanning using the Cirrus HD-OCT optic disc cube $200 \times 200$ protocol and 3 sessions of macular scanning using the macular cube $516 \times 258$ protocol. The peripapillary scan allowed measurement of RNFL thickness and ONH topographic parameters, whereas the macular scan allowed determination of macular GCIPL thickness using the GCA algorithm. Detailed descriptions of how the Cirrus HD-OCT peripapillary GCA, RNFL, and ONH algorithms operate have been presented elsewhere. ${ }^{5,14,15}$ Only scans with a signal strength of 6 or more and without motion (blinking or saccades) artifacts, segmentation failure caused by algorithm dysfunction, vitreous floaters, or other causes were kept for analysis. Parameters included in the analysis were average and sectoral (superior, superonasal, inferonasal, inferior, inferotemporal, superotemporal) for GCIPL thicknesses; average, quadrant (temporal, superior, nasal, inferior), and clock hours for RNFL thickness; as well as rim area, vertical cup-to-disc ratio (VCDR), and average cupto-disc ratio for $\mathrm{ONH}$ parameters.

\section{Data Analysis}

The Cirrus HD-OCT GCIPL, RNFL, and ONH significance maps use the same 3-level color coding system to determine whether the measurement is within normal (green), borderline (yellow), or outside normal range (red). In other words, green, yellow, and red indicate that the measurement has $5 \%$ to $95 \%, 1 \%$ to $5 \%$, and less than $1 \%$ probability to be within normal range for an age-matched normal population, respectively. The GCIPL and RNFL scans were classified as abnormal if at least 2 of the 3 scans had the average or at least 1 sector flagged at $1 \%$ to $5 \%$ (yellow) or less than $1 \%$ (red). For $\mathrm{ONH}$ analysis, scans were considered abnormal if rim area, cup-to-disc ratio, or VCDR were flagged at $1 \%$ to $5 \%$ or at less than $1 \%$ significance level on at least 2 of the 3 scans. Although 3 scans were acquired per eye, only thickness values from the first of the 3 scans, if they were all abnormal or normal, or from the first of the 2 abnormal or normal of 3 scans, were used in statistical analyses. Sensitivity, specificity, positive likelihood ratio (PLR), and negative likelihood ratio (NLR) were calculated for each GCIPL, RNFL, and ONH parameter. These diagnostic performance measures also were determined after combining the best GCIPL with the best RNFL or ONH parameters using the binary or-logic and and-logic methods. For this study, the likelihood ratio was interpreted using McGee's scale ${ }^{16}$ that we modified slightly for better understanding: (1) PLR (likelihood of disease) >10, strong; 5-10, moderate; 2-4.9, fair; and >1-1.9, low; (2) LR of 1, no diagnostic value; and (3) NPR (likelihood of disease absence) of $0.5-0.9$, low; 0.2-0.49, fair; $0.1-0.19$, moderate; and $<0.1$, strong. The or-logic approach requires at least one of the parameters in consideration to be abnormal, whereas the and-logic method requires all parameters in the model to be abnormal for the combination to be considered abnormal and suggestive of glaucoma. Sensitivities and specificities were compared with the chi-square test. Comparison of continuous variables between groups was determined using the Student $t$ test. Statistical analyses were performed with SPSS version 21.0 (SPSS, Inc, Chicago, IL) and MedCalc version 11.1.0 (MedCalc Software bvba, Mariakerke, Belgium). A $P$ value of less than 0.05 was used to indicate a statistically significant difference.

\section{Results}

Participants' mean age was $63.1 \pm 9.1$ years (range, $45.6-83.0$ years) for normal subjects and $66.4 \pm 10.8$ years (range, $45.8-89.3$ years) for glaucoma patients $(P=0.095)$. There were 31 women and 18 men in the normal group $(\mathrm{n}=49)$ and 28 women and 22 men in the glaucoma group $(\mathrm{n}=50)$. The average visual field mean deviation of glaucomatous patients was $-2.96 \pm 1.93 \mathrm{~dB}$. The mean spherical equivalent was similar in normal subjects $(-0.40 \pm 1.71 \mathrm{D})$ and glaucomatous patients $(-0.42 \pm 1.86 \mathrm{D} ; P=0.95)$.

The comparison between the 2 groups with regard to RNFL, ONH, and GCIPL parameters studied is given in Table 1. All RNFL (except RNFL at clock hours 3, 4, and 9; RNFL clock hours are not listed in Table 1) and GCIPL parameters were significantly thinner in 
Table 1. Ganglion Cell-Plexiform Layer Thickness, Retinal Nerve Fiber Layer Thickness and Optic Nerve Head Measurements in Normal and Glaucomatous Subjects

\begin{tabular}{lccr}
\hline \multicolumn{1}{c}{ Parameter } & Normal & Glaucoma & P Value \\
\hline GCIPL $(\mu \mathrm{m})$ & & & \\
Average & $78.2 \pm 8.3$ & $69.4 \pm 6.9$ & $<0.001$ \\
Minimum & $75.9 \pm 11.3$ & $61.3 \pm 9.2$ & $<0.001$ \\
Superotemporal & $78.7 \pm 7.9$ & $69.7 \pm 6.9$ & $<0.001$ \\
Superior & $79.8 \pm 8.4$ & $72.8 \pm 8.3$ & $<0.001$ \\
Superonasal & $80.1 \pm 8.8$ & $74.7 \pm 9.0$ & 0.004 \\
Inferonasal & $76.6 \pm 8.8$ & $70.1 \pm 9.3$ & 0.001 \\
Inferior & $75.2 \pm 9.8$ & $64.9 \pm 9.3$ & $<0.001$ \\
Inferotemporal & $78.9 \pm 8.6$ & $64.7 \pm 9.1$ & $<0.001$ \\
RNFL ( $\mu$ m) & & & \\
Average & $89.7 \pm 9.0$ & $70.5 \pm 10.6$ & $<0.001$ \\
Superior & $109.9 \pm 15.7$ & $83.1 \pm 14.2$ & $<0.001$ \\
Inferior & $116.2 \pm 15.1$ & $80.7 \pm 18.9$ & $<0.001$ \\
Temporal & $64.1 \pm 13.4$ & $56.0 \pm 10.0$ & 0.001 \\
Nasal & $68.4 \pm 10.6$ & $62.2 \pm 12.0$ & 0.008 \\
ONH measurements & & & \\
CDR & $0.47 \pm 0.1$ & $0.70 \pm 0.1$ & $<0.001$ \\
VCDR & $0.45 \pm 0.1$ & $0.72 \pm 0.1$ & $<0.001$ \\
Rim area $(\mu \mathrm{m})$ & $1.26 \pm 0.2$ & $0.88 \pm 0.2$ & $<0.001$ \\
& & &
\end{tabular}

$\mathrm{CDR}=$ cup-to-disc area ratio; GCIPL = ganglion cell-inner plexiform layer; $\mathrm{ONH}=$ optic nerve head; $\mathrm{RNFL}=$ retinal nerve fiber layer; VCDR = vertical cup-to-disc diameter ratio.

the glaucoma group compared with the control group. Glaucomatous patients had a significantly thinner rim area and significantly larger cup-to-disc ratio and VCDR compared with controls.

The sensitivity, specificity, PLR, and NLR for each parameter studied are shown in Table 2 (RNFL clock hours not listed). For single GCIPL parameters, the minimum was the best discriminant with $82 \%$ sensitivity and $87.8 \%$ specificity, followed by the inferotemporal sector (70\% and $87.8 \%$, respectively). The other GCIPL parameters had low sensitivities ranging between $24 \%$ and $60 \%$. The diagnosis based on at least 1 abnormal GCIPL parameter yielded sensitivity and specificity values of $88 \%$ and $81.6 \%$, respectively, which was a significant increase in sensitivity relative to average and single GCIPL sectoral parameters. Among single RNFL parameters, the inferior quadrant outperformed the other parameters with sensitivity and specificity values of $74 \%$ and $95.9 \%$, respectively, followed by average RFNL (64\% and $95.9 \%$, respectively), clock hour 7 (64\% and $91.8 \%$, respectively), clock hour $6(64 \%$ and $89.8 \%$, respectively), and superior quadrant $(62 \%$ and $89.8 \%$, respectively). All other RNFL parameters had a sensitivity range of $10 \%$ to $48 \%$ and specificity range of $87.8 \%$ to $100 \%$. If glaucoma detection was based on any abnormal RNFL quadrant, the sensitivity increased to $86 \%$, whereas the specificity decreased to $79.6 \%$. A similar tradeoff between sensitivity (92\%) and specificity $(59.2 \%)$ was observed when any abnormal clock hour sector was considered as glaucoma detection criteria. Detection based on at least 1 abnormal RNFL parameter yielded a sensitivity of $86 \%$ and specificity of $77.6 \%$. No significant differences were observed in all diagnostic measures between the minimum GCIPL, inferior quadrant RNFL, and rim area $(P=$ $0.26-0.76$ for sensitivities and $P=0.14-0.98$ for specificities). The sensitivity and specificity based on at least 1 abnormal ONH parameter were $72 \%$ and $95.9 \%$, respectively. All $3 \mathrm{ONH}$ parameters, inferior quadrant, and average RNFL conferred strong PLRs for early glaucoma, whereas minimum and inferotemporal GCIPL were associated with moderate PLRs. The NLR was fair (range, 0.21-0.46) for all of these parameters.

Table 3 shows the diagnostic performance obtained using both the or-logic and and-logic after considering only averages and best RNFL and GCIPL parameters. In general, or-logic approaches provided better diagnostic performances than and-logic scenarios. The best diagnostic performances were provided by or-logic combinations of minimum GCIPL and average RNFL (sensitivity, 94\%; specificity, $85.7 \%$ ), minimum GCIPL and rim area (sensitivity, 92\%; specificity, 85.7\%), and minimum GCIPL and

Table 2. Diagnostic Performance of Ganglion Cell-Plexiform Layer, Retinal Nerve Fiber Layer, and Optic Nerve Head Parameters in Early Glaucoma

\begin{tabular}{lcccc}
\hline Parameter & $\begin{array}{c}\text { Sensitivity } \\
\text { (95\% Confidence Interval) }\end{array}$ & $\begin{array}{c}\text { Specificity } \\
\text { (95\% Confidence Interval) }\end{array}$ & $\begin{array}{c}\text { Positive Likelihood Ratio } \\
\text { (95\% Confidence Interval) }\end{array}$ & $\begin{array}{c}\text { Negative Likelihood Ratio } \\
\text { (95\% Confidence Interval) }\end{array}$ \\
\hline GCIPL & & & & \\
Minimum & $82.0(68.1-90.9)$ & $87.8(74.5-94.9)$ & $6.69(3.13-14.33)$ & $0.21(0.11-0.37)$ \\
IT & $70.0(55.2-81.7)$ & $87.8(74.5-94.9)$ & $5.72(2.64-12.36)$ & $0.34(0.22-0.52)$ \\
Inferior & $60.0(45.2-73.3)$ & $89.8(77.0-96.2)$ & $5.88(2.49-13.91)$ & $0.45(0.32-0.63)$ \\
ST & $52.0(37.6-66.1)$ & $85.7(72.1-93.6)$ & $3.64(1.74-7.60)$ & $0.56(0.42-0.75)$ \\
Average & $48.0(33.9-62.4)$ & $85.7(72.1-93.6)$ & $3.36(1.60-7.07)$ & $0.61(0.46-0.80)$ \\
IN & $42.0(28.5-56.7)$ & $87.8(74.5-94.9)$ & $3.43(1.51-7.77)$ & $0.66(0.52-0.84)$ \\
Superior & $40.0(26.7-54.8)$ & $87.8(74.5-94.9)$ & $3.27(1.43-7.44)$ & $0.68(0.54-0.86)$ \\
SN & $24.0(13.5-38.5)$ & $87.8(74.5-94.9)$ & $1.96(0.79-4.81)$ & $0.87(0.74-1.02)$ \\
RNFL & & & & \\
Inferior & $74.0(59.4-84.9)$ & $95.9(84.9-99.3)$ & $18.13(4.62-71.15)$ & $0.27(0.17-0.43)$ \\
Average & $64.0(49.1-76.7)$ & $95.9(84.9-99.3)$ & $15.68(3.79-61.9)$ & $0.37(0.26-0.54)$ \\
Superior & $62.0(47.2-75.0)$ & $89.8(77.0-96.2)$ & $6.08(2.58-14.34)$ & $0.42(0.29-0.61)$ \\
Nasal & $18.0(9.05-31.9)$ & $98.0(87.8-99.9)$ & $8.82(1.16-67.03)$ & $0.83(0.73-0.95)$ \\
Temporal & $16.0(7.64-29.7)$ & $93.9(82.1-98.4)$ & $2.61(0.73-9.28)$ & $0.89(0.79-1.01)$ \\
ONH & & & & \\
Rim area & $68.0(53.2-80.1)$ & $98.0(87.8-99.9)$ & $33.3(4.74-233.9)$ & $0.33(0.22-0.49)$ \\
VCDR & $68.0(53.2-80.1)$ & $98.0(87.8-99.9)$ & $33.3(4.74-233.9)$ & $0.33(0.22-0.49)$ \\
CDR & $54.0(39.5-67.9)$ & $100(90.9-100)$ & Infinity & $0.46(0.34-0.62)$
\end{tabular}

$\mathrm{CDR}=$ cup-to-disc area ratio; GCIPL $=$ ganglion cell-inner plexiform layer; IN $=$ inferonasal; IT $=$ inferotemporal; ONH $=$ optic nerve head; RNFL $=$ retinal nerve fiber layer; $\mathrm{SN}=$ superonasal; ST = superotemporal; VCDR = vertical cup-to-disc diameter ratio. 
Table 3. Diagnostic Performance Based on Or-Logic and And-Logic Combinations of Ganglion Cell-Plexiform Layer with Retinal Nerve Fiber Layer or Optic Nerve Head Parameters in Early Glaucoma

\begin{tabular}{lcccc}
\hline \multicolumn{1}{c}{ Parameter } & $\begin{array}{c}\text { Sensitivity } \\
\text { (95\% Confidence } \\
\text { Interval) }\end{array}$ & $\begin{array}{c}\text { Specificity } \\
\text { (95\% Confidence } \\
\text { Interval) }\end{array}$ & $\begin{array}{c}\text { Positive Likelihood } \\
\text { Ratio (95\% Confidence } \\
\text { Interval) }\end{array}$ & $\begin{array}{c}\text { Negative Likelihood } \\
\text { Ratio (95\% Confidence } \\
\text { Interval) }\end{array}$ \\
\hline Minimum GCIPL or average RNFL & $94.0(82.5-98.4)$ & $85.7(72.1-93.6)$ & $6.58(3.30-13.11)$ & $0.07(0.02-0.21)$ \\
Minimum GCIPL or rim area & $92.0(79.9-97.4)$ & $85.7(72.1-93.6)$ & $6.44(3.23-12.85)$ & $0.09(0.04-0.24)$ \\
Minimum GCIPL or inferior RNFL & $92.0(79.9-97.4)$ & $83.7(69.8-92.2)$ & $5.64(2.97-10.68)$ & $0.09(0.04-0.25)$ \\
IT GCIPL or inferior RNFL & $86.0(72.6-93.7)$ & $83.7(69.8-92.2)$ & $5.27(2.77-10.03)$ & $0.17(0.08-0.34)$ \\
Average GCIPL or average RNFL & $76.0(61.5-86.5)$ & $79.6(65.2-89.3)$ & $3.72(2.09-6.61)$ & $0.30(0.18-0.50)$ \\
Any GCIPL or any RNFL & $96.0(85.1-99.3)$ & $63.3(48.3-76.2)$ & $2.61(1.80-3.79)$ & $0.06(0.02-0.25)$ \\
Any GCIPL or any ONH & $94.0(82.5-98.4)$ & $77.6(63.0-87.8)$ & $4.19(2.48-7.08)$ & $0.08(0.03-0.23)$ \\
Any GCIPL and any RNFL & $78.0(63.7-88.0)$ & $95.9(84.9-99.3)$ & $19.11(4.88-74.85)$ & $0.23(0.14-0.39)$ \\
Any GCIPL and any ONH & $68.0(53.2-80.1)$ & $100(90.9-100)$ & Infinity & $0.32(0.21-0.48)$ \\
Minimum GCIPL and inferior RNFL & $64.0(49.1-76.7)$ & $100(90.9-100)$ & Infinity & $0.36(0.25-0.52)$ \\
IT GCIPL and inferior RNFL & $60.0(45.2-73.3)$ & $100(90.9-100)$ & Infinity & $0.40(0.28-0.56)$ \\
Minimum GCIPL and rim area & $58.0(43.3-71.5)$ & $100(90.9-100)$ & Infinity & $0.42(0.30-0.58)$ \\
Minimum GCIPL and average RNFL & $52.0(37.6-66.1)$ & $98.0(87.8-99.9)$ & $25.48(3.60-180.54)$ & $0.49(0.37-0.65)$ \\
Average GCIPL and average RNFL & $34.0(21.6-48.9)$ & $98.0(87.8-99.9)$ & $16.66(2.31-120.41)$ & $0.67(0.55-0.82)$
\end{tabular}

$\mathrm{GCIPL}=$ ganglion cell-inner plexiform layer; IT $=$ inferotemporal; $\mathrm{ONH}=$ optic nerve head; RNFL $=$ retinal nerve fiber layer.

inferior quadrant RNFL (sensitivity, 92\%; specificity, 83.7\%). Combining any abnormal GCIPL with any abnormal RNFL or ONH parameter by the or-logic method achieved sensitivities of $96 \%$ and $94 \%$, respectively, for corresponding specificities of $63.6 \%$ and $77.6 \%$. The sensitivities and specificities of the 3 best or-logic combinations did not differ significantly among them $(P=$ 0.96-1.00); however, the sensitivities were higher than that of the best and-logic combination, despite not reaching the significance level $(P=0.06-0.13)$. These 3 best or-logic combinations conferred moderate PLRs and strong NLRs for early glaucoma, whereas most and-logic combinations were associated with strong PLRs and low to fair NLRs.

\section{Discussion}

The diagnosis of glaucoma in its early stages is challenging and one of the major obstacles to fighting glaucoma is the failure to identify individuals with the condition in the early stages until significant functional loss has occurred. Thus, detection of early glaucoma allows early treatment to delay loss of vision. Efforts to detect glaucoma early have resulted in recent years in the development of new imaging methods. One of these methods is spectral-domain OCT, the introduction of which has enabled identification of new structural parameters through novel quantitative methods for measuring either the entire retinal thickness or inner retinal layers in the macular region. ${ }^{15,17,18}$ The recent renewed interest in measuring macular layers for diagnosing and monitoring glaucoma has resulted from the development of powerful segmentation algorithms, such as the Cirrus-OCT ganglion cell analysis, which calculates the thickness of the macular GCIPL. ${ }^{15}$ We first showed that GCIPL thickness is a good indicator of glaucoma and that its glaucoma diagnostic performance is similar to that of RNFL. ${ }^{4}$ Subsequent studies by other groups ${ }^{8,9,19,20}$ built on this work to confirm these findings and to show the usefulness of GCIPL for glaucoma diagnosis. In this study, we investigated whether combining GCIPL, ONH, and RNFL parameters optimizes the diagnostic performance for early glaucoma compared with single GCIPL and RNFL parameters.

We found that compared with GCIPL, ONH, and RNFL parameters used individually, combining them using the orlogic method enhances the diagnostic performance for early glaucoma. For example, pairing the minimum GCIPL and average RNFL or the minimum GCIPL and rim area using the or-logic method improved the sensitivity, NPV, and NLR compared with the best single GCIPL, RNFL, or ONH parameters, without significantly affecting the specificity. Our results are similar to those reported recently by Jeoung et $\mathrm{al}^{20}$ with regard to the above first combination. However, there are 3 main differences between that study and ours. First, rather than combining the inferior GCIPL sectors and superior GCIPL sectors to form the inferior and superior quadrant GCIPL, respectively, we chose not to combine GCIPL sectors to avoid masking the performance of individual sectors. Indeed, our finding that the or-logic combination of inferotemporal sector alone with inferior quadrant RNFL (Table 3) resulted in a significant increase of sensitivity while maintaining good specificity and NLR may suggest that it may not be necessary to combine GCPIL sectors to improve their diagnostic performance. Second, we combined GCIPL and RNFL parameters with best diagnostic performances rather than combining parameters based on similarity of their locations in the peripapillary and macular regions. Doing so allowed achieving higher diagnostic performances than those reported by Jeoung et al. ${ }^{20}$ Third, scanning of the $\mathrm{ONH}$ using spectral-domain OCT has improved the clinician's approach to the optic disc assessment by providing detailed and reliable quantitative information beyond qualitative and subjective clinical examination. Because structural changes to the $\mathrm{ONH}$ are a hallmark of glaucoma and may precede detection of perimetric damage, the diagnostic performance of GCIPL may be optimized if used in combination with $\mathrm{ONH}$ assessment. We therefore also evaluated the performance after combining GCIPL and ONH parameters, an 
aspect that was not addressed in Jeoung et al. ${ }^{20}$ Again, the or-logic combination of minimum GCIPL and rim area resulted in a diagnostic performance equivalent to that of the or-logic combination of minimum GCIPL and average RNFL thickness, but better than corresponding and-logic combination. It is worth noting that the minimum GCIPL consistently is not only the best single GCIPL parameter across studies in terms of diagnostic performance, ${ }^{4,9,20}$ but also seems to be the most influential parameter in or-logic combinations.

And-logic combinations of GCIPL and RNFL parameters resulted in low diagnostic performances for early glaucoma both in our study and in a recent report, ${ }^{20}$ indicating that it is a poor approach for the diagnosis of early glaucoma. Interestingly, the and-logic combination also was found to generate low sensitivities even for moderate to severe glaucoma. $^{20}$ This finding may be the result of the low agreement between abnormal GCIPL and abnormal RNFL on Cirrus OCT. For example, the agreement $(\kappa)$ between average GCIPL and average RNFL was only 0.34 , which supports the results of our earlier study. ${ }^{21}$ This lack of agreement between RNFL and GCIPL parameters is exemplified further in Table 2 by the difference in PLRs between average GCIPL (fair), average RNFL (strong), and their NLRs (strong for average GCIPL and fair for average RNFL). The clinical relevance of this finding is that the diagnosis of early glaucoma should be based on either abnormal GCIPL or RNFL parameters, not necessarily on both abnormal GCIPL and RNFL parameters. Ultimately, the finding also suggests that the Cirrus GCA has been a valuable complementary diagnostic tool to RNFL analysis.

Two prior studies also used software-provided classification codes to determine the diagnostic performances of glaucoma-related structural parameters measured with spectral-domain OCT. Garas et $\mathrm{al}^{12}$ reported sensitivities not exceeding $69.6 \%$ (average) for RNFL parameters, 72\% (focal loss volume) for ganglion cell complex parameters, and $84.1 \%$ (VCDR) for ONH parameters. However, these values were based on a study population with early to severe glaucoma, suggesting lower sensitivities for early glaucoma alone. Chang et $\mathrm{al}^{11}$ studied glaucoma patients with early to moderate functional deficit and found sensitivities of $83 \%$ and $65 \%$ for average RNFL with corresponding specificities of $88 \%$ and $100 \%$ for borderline and outside normal limits measurements, respectively. Values for early glaucoma were not provided separately, making the comparison with our study difficult. None of these 2 studies reported PLRs or NLRs.

From a methodologic standpoint, we classified a subject as having an abnormal scan if 2 of the 3 RNFL or GCIPL scans had borderline measurements or if measurements fell outside normal limits, because conceptually both are the result of structural loss. Compared with other studies where only 1 scan was acquired per patient, ${ }^{12,20}$ our method is likely to reduce the rate of false-positive and false-negative scans and therefore to provide reliable values of diagnostic measures.

We acknowledge that our study has limitations. The sample size is small and any attempt to generalize the results should be made with caution. However, it is also important to note that similar results were found using a study population 3 times as large. ${ }^{20}$ In addition, because OCTgenerated probability of abnormality is based on a comparison of measurements to built-in normative database values, one would expect measurements obtained in some normal subjects to be borderline. ${ }^{22}$ Thus, some of the normal subjects in our study population might have been classified falsely as abnormal as a result of their values being closer to the lower limit of the normal range.

In conclusion, the results of this study confirm that minimum GCIPL has the highest diagnostic performance for early perimetric glaucoma among all GCIPL parameters and that its performance is similar to that of the best RNFL and ONH parameters. The binary combination of minimum GCIPL and average RNFL or minimum GCIPL and rim area using the or-logic approach improves the glaucoma diagnostic performance compared with and-logic combinations of GCIPL and RNFL parameters, as well as single GCIPL or RNFL parameters. This finding may be clinically valuable for the diagnosis of early glaucoma.

\section{References}

1. Arintawati $\mathrm{P}$, Sone $\mathrm{T}$, Akita $\mathrm{T}$, et al. The applicability of ganglion cell complex parameters determined from SD-OCT images to detect glaucomatous eyes [report online]. J Glaucoma. Available at: http://ovidsp.tx.ovid.com. Accessed September 26, 2013.

2. Kim NR, Hong S, Kim JH, et al. Comparison of macular ganglion cell complex thickness by Fourier-domain OCT in normal tension glaucoma and primary open-angle glaucoma. J Glaucoma 2013;22:133-9.

3. Kim NR, Lee ES, Seong GJ, et al. Comparing the ganglion cell complex and retinal nerve fibre layer measurements by Fourier domain OCT to detect glaucoma in high myopia. Br J Ophthalmol 2011;55:1115-21.

4. Mwanza JC, Durbin MK, Budenz DL, et al. Glaucoma diagnostic accuracy of ganglion cell-inner plexiform layer thickness: comparison with nerve fiber layer and optic nerve head. Ophthalmology 2012;119:1151-8.

5. Mwanza JC, Oakley JD, Budenz DL, Anderson DR; Cirrus Optical Coherence Tomography Normative Database Study Group. Ability of Cirrus HD-OCT optic nerve head parameters to discriminate normal from glaucomatous eyes. Ophthalmology 2011;118:241-8.

6. Schulze A, Lamparter J, Pfeiffer N, et al. Diagnostic ability of retinal ganglion cell complex, retinal nerve fiber layer, and optic nerve head measurements by Fourier-domain optical coherence tomography. Graefes Arch Clin Exp Ophthalmol 2011;249:1039-45.

7. Curcio CA, Allen KA. Topography of ganglion cells in human retina. J Comp Neurol 1990;300:1-25.

8. Kotowski J, Folio LS, Wollstein G, et al. Glaucoma discrimination of segmented Cirrus spectral domain optical coherence tomography (SD-OCT) macular scans. Br J Ophthalmol 2012;96:1420-5.

9. Takayama K, Hangai M, Durbin M, et al. A novel method to detect local ganglion cell loss in early glaucoma using spectraldomain optical coherence tomography. Invest Ophthalmol Vis Sci 2012;53:6904-13.

10. Budenz DL, Michael A, Chang RT, et al. Sensitivity and specificity of the StratusOCT for perimetric glaucoma. Ophthalmology 2005;112:3-9. 
11. Chang RT, Knight OJ, Feuer WJ, Budenz DL. Sensitivity and specificity of time-domain versus spectral-domain optical coherence tomography in diagnosing early to moderate glaucoma. Ophthalmology 2009;116:2294-9.

12. Garas A, Vargha P, Hollo G. Diagnostic accuracy of nerve fibre layer, macular thickness and optic disc measurements made with the RTVue-100 optical coherence tomograph to detect glaucoma. Eye (Lond) 2011;25:57-65.

13. Hodapp E, Parrish RK II, Anderson DA. Clinical decisions in glaucoma. St. Louis, MO: Mosby-Year Book; 1993:52-61.

14. Mwanza JC, Chang RT, Budenz DL, et al. Reproducibility of peripapillary retinal nerve fiber layer thickness and optic nerve head parameters measured with Cirrus HD-OCT in glaucomatous eyes. Invest Ophthalmol Vis Sci 2010;51:5724-30.

15. Mwanza JC, Oakley JD, Budenz DL, et al. Macular ganglion cell-inner plexiform layer: automated detection and thickness reproducibility with spectral domain-optical coherence tomography in glaucoma. Invest Ophthalmol Vis Sci 2011;52:8323-9.

16. McGee S. Simplifying likelihood ratios. J Gen Intern Med 2002;17:646-9.
17. Asrani S, Rosdahl JA, Allingham RR. Novel software strategy for glaucoma diagnosis: asymmetry analysis of retinal thickness. Arch Ophthalmol 2011;129:1205-11.

18. Tan O, Chopra V, Lu AT, et al. Detection of macular ganglion cell loss in glaucoma by Fourier-domain optical coherence tomography. Ophthalmology 2009;116:2305-14.

19. Akashi A, Kanamori A, Nakamura M, et al. Comparative assessment for the ability of Cirrus, RTVue and 3D-OCT to diagnose glaucoma. Invest Ophthalmol Vis Sci 2013;54:4478-84.

20. Jeoung JW, Choi YJ, Park KH, Kim DM. Macular ganglion cell imaging study: glaucoma diagnostic accuracy of spectraldomain optical coherence tomography. Invest Ophthalmol Vis Sci 2013;54:4422-9.

21. Mwanza JC, Sayyad FE, Aref AA, Budenz DL. Rates of abnormal retinal nerve fiber layer and ganglion cell layer OCT scans in healthy myopic eyes: Cirrus versus RTVue. Ophthalmic Surg Lasers Imaging 2012;43(suppl):S67-74.

22. Budenz DL, Anderson DR, Varma R, et al. Determinants of normal retinal nerve fiber layer thickness measured by Stratus OCT. Ophthalmology 2007;114:1046-52.

\section{Footnotes and Financial Disclosures}

Originally received: August 9, 2013.

Final revision: October 27, 2013.

Accepted: October 28, 2013

Available online: January 6, 2014.

Manuscript no. 2013-1325.

${ }^{1}$ Department of Ophthalmology, University of North Carolina School of Medicine, Chapel Hill, North Carolina.

${ }^{2}$ Glaucoma Associates of Texas, Dallas, Texas.

${ }^{3}$ Bascom Palmer Eye Institute, University of Miami Miller School of Medicine, Miami, Florida.

${ }^{4}$ Department of Ophthalmology, Stanford University School of Medicine, Palo Alto, California.
Financial Disclosure(s):

The author(s) have no proprietary or commercial interest in any materials discussed in this article.

Supported by an unrestricted grant from Research to Prevent Blindness, Inc, New York, New York.

Correspondence:

Donald L. Budenz, MD, MPH, Department of Ophthalmology, University of North Carolina at Chapel Hill, 5151 Bioinformatics Building, CP no. 7040, 130 Mason Farm Road, Chapel Hill, NC 27599. E-mail: donald_budenz@med.unc.edu. 